\title{
Analysis on Teaching Innovation of Operations Research Course Under the Background of New Business in the Era of Big Data
}

\begin{abstract}
Zhang Linlan ${ }^{1, a}$
${ }^{1}$ School of Business, Hubei University, Wuhan, Hubei, China

amisalinlan9904@163.com

ABSTRACT

The year of 2021 has entered the era of big data artificial intelligence, and new challenges to the traditional business model are flourishing. How to deal with the challenges in the era of big data and the requirements of new business science is the primary task of current operational research teaching innovation. At present, most of the operational research teaching in universities adopts the general teaching materials and teaching mode, which cannot reflect the characteristics of The Times. This paper analyzes the existing problems in operational research teaching and puts forward some innovative ideas. It is hoped that it will be helpful to the current operational research teaching in universities and promote the cultivation of applied talents in universities.
\end{abstract}

Keywords: Operations Research, teaching models, big data, new business

\section{大数据时代新商科背景下运筹学课程教学创新探析}

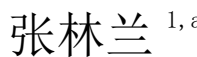

\author{
${ }^{1}$ 湖北大学商学院, 武汉, 湖北, 中国 \\ amisalinlan9904@163.com
}

\begin{abstract}
摘要
2021 年已进入大数据人工智能时代，对传统的商科模式提出了新挑战。如何应对大数据时代的挑战和新商科 的要求, 是当前运筹学教学创新的首要任务。目前大多数大学运筹学教学采用通用的教材和教学模式, 体现不 了专业的时代特点。本文分析了当前运筹学教学中存在的问题, 提出了几点创新思路。希望能对当前的大学运 筹学教学有所帮助, 促进大学应用型人才的培养。
\end{abstract}

关键词: 运筹学, 教学模式, 大数据, 新商科

1. 前言

运筹学是一门研究如何实现最优安排的学科。 世界公认运筹学起源于第二次世界大战期间英、美等 国军事部门成立的一些研究小组的研究活动 [1,2]。 运筹学英文翻译为 Operational Research, 英文原 意是运用研究或作战研究。在中国古代很多典故里就 蕴含了运筹学思想, 比如 《孙子兵法》, 《田忌赛马》, 《丁渭修宫》, 《沈括运粮》等。我国的运筹学思想在 国际上也得到了承认和认可。1981 年美国军事运筹
学会出版了一本 “Systems analysis and modeling in defense” , 书中称 “孙武是世界上第一个军事运 筹学的实践家”。运筹学在战争中的成功吸引了更多 的资源投入这一研究领域。二战结束后, 经济发展成 为各方注视的焦点, 经济和工业界很多问题都可以用 运筹学方法来解决。而计算机的出现为运筹学的应用 提供了最好的技术支持。尤其是近年来, 人工智能发 展迅速, 大数据时代已然到来。利用大数据技术和人 工智能手段, 运筹学可以发挥更广阔的作用, 提供更 先进的决策方案。比如, 2016 年和 2017 年的围棋人 机大战, 谷歌公司开发的围棋人工智能程序 “阿尔法 
围棋”（AlphaGo）分别战胜了韩国围棋九段棋手李 世石和中国围棋九段棋手柯洁。在 “阿尔法围棋” 程 序里就大量运用了运筹学里的动态规划和决策树的 方法。“人工智能”这个概念逐渐被人们熟悉和广泛 关注。

近年来, 作为基于数据的优化工具和学科, 运筹 学这一课程获得了前所未有的广泛关注, 在商科和工 科等应用学科各专业都开设了这一课程。大数据、云 计算、人工智能、共享经济等新兴技术的迅猛发展, 使得传统的商业模式已经由实体店走向互联网电商, 融合线上和线下双发展。新商业模式创新已然成为未 来商业服务创新发展的重头。在现有商科的发展基础 上, 新商科承受了更多来自科技、社会、经济所带来 的新挑战, 新一轮的科技革命和产业革命已经开始。 新商科一定要根据实体经济供给侧的需求, 结合线上 和线下两种经营模式, 走市场化、企业化的合作之路 [3]。作为大学众多专业课程中的重要基础课程, 运 筹学在教学过程中还存在诸多问题。当前的运筹学课 程教学内容, 主要集中在优化经济管理领域的现实生 活遇到的问题, 缺乏计算机和人工智能领域方面的运 用, 体现不了大数据时代和新商科的特点 $[4,5]$ 。本 文基于大数据时代的背景, 对新商科运筹学的课程教 学模式和教学方法等进行初步探讨, 提出一些建议。

\section{2. 运筹学教学存在的问题}

运筹学作为各大学新商科管理类专业课程体系 中的核心基础必修课程, 已积累了多年的教学经验和 成果。运筹学课程理论性很强, 需要扎实的数学基础, 尤其是线性代数。如果同学们对线性代数的知识掌握 不牢固的话, 学起运筹学就特别难以理解, 学起来很 困难。多年来, 为了强调运筹学的应用性, 在课堂教 学中尽量弱化理论知识的讲解, 尤其是定理的数学证 明。但是, 忽略学习理论证明会导致学生对运筹学方 法理论的理解不深刻, 不知道为什么要这样做。尤其 是大多数学校运筹学课程的课时一般都为 48 课时, 学时有限。往往只能选讲部分内容, 比如线性规划与 单纯形法, 线性目标规划, 整数线性规划和动态规划 等。课时的限制, 加上学生对数学的畏难情绪, 尤其 是随着时代的发展, 在运筹学教学过程中仍存在一些 函待解决的问题。

(一)、学生对课程认识不深刻, 对学习运筹学 的用处了解不全面。运筹学是一门使用最优化理论与 决策分析方法分析实际问题, 找出最优解决方案的学 科。主要用处是给现实生活各行各业的决策者提供理 论参考和决策建议。1956 年, 在钱学森、许国志等 老一辈运筹学家的积极推动下, 我国成立了第一个运 筹学研究小组 $[6,7]$ 。1958 年, 管梅谷先生提出了被 国际运筹学教材赋名的中国邮递员问题, 使得我国运 筹学的研究在国际运筹学领域占有一席之地。我国运 筹学经过多年的发展, 取得了长足的进步和成果, 但 是仍然与以西方国家为代表的国际运筹学先进研究 水平有较大的差距。尤其是当前运筹学的教学学时有
限, 大多停留在理论教学层面。学生学起来比较难以 理解, 也更难体会到运筹学在实际生活中的应用了。 我国新时代大学生应认识到学好运筹学, 不仅仅是解 决实际问题, 更是要赶上并超越西方国家, 是时代赋 予他们的使命。

(二)、教学内容过于理论偏数学化, 案例分析 较少且偏陈旧, 缺乏软件求解, 不能紧跟大数据和人 工智能发展的特点。运筹学是管理类专业的大学生必 须掌握的专业基础必修课。课程理论性很强, 很多原 理和方法的推导需要扎实的数学基础, 尤其是要用到 线性代数的理论。在学习过程中, 为了加深对运筹学 优化方法的理解, 不可避免的要学习优化方法背后的 数学证明推导。由于课时有限, 学生对理论推导学起 来普遍困难。如果不讲理论, 直接使用优化方法求解。 往往会导致学生对运筹学优化方法知其然, 不知其所 以然。忽视理论知识的学习, 会导致学生对运筹学的 优化方法掌握不牢固。尽管教师在教学过程中, 弱化 了数学推导过程, 但是为了让学生掌握运筹学的优化 方法, 仍要花大量课时讲解模型建模和求解。管理类 专业运筹学课时大多为 48 学时, 课时的限制导致学 生没有多余的时间来学习软件求解运筹学优化问题。 在我国当前管理类专业中, 运筹学虽然是一门管理学 科的基础课, 但往往给学生的感觉是一门数学课。学 生学起来觉得是一门纯理论的学科, 不能认识到运筹 学在管理实践中的运用, 学生的实践能力得不到提高。

(三)、教学手段偏传统, 主要以教师讲授为主。 由于课程理论性强, 学生学起来比较吃力, 主要以课 前预习, 课堂教师讲解, 课后学生练习为主。学时的 限制导致教师只能在有限的课堂时间内讲解重要的 基础方法, 忽略了培养学生主动思考, 利用软件求解 模型的能力。比如, 运筹学的第一章就是讲解单纯形 法。单纯形法的原理要结合实际的优化问题来讲解。 大多数学生都能接受。但是单纯形的计算和迭代过程, 对于学生来说往往计算量很大。实际上, 可以通过学 习软件来应用单纯形求解。这样既锻炼了学生的实践 能力, 又能让学生理解理论在实践中的应用。但是学 时的限制, 和学校实验设施的有限, 往往大多数学校 都没有开设实验上机课, 只有单纯的理论教学。教材 内容偏于理论化, 缺乏实践问题的练习。对于基础知 识薄弱的学生, 学起来难以接受理解, 越学越觉得困 难, 从而导致没有学习运筹学的热情, 学习积极性也 不高。

(四)、课程考核方式单一。目前大多数大学对 于运筹学的课程考核, 主要采取平时成绩和期末考试 加权的考核方式。平时成绩包括考勤, 课堂表现和课 后作业等, 期末考试主要是闭卷考试, 重点考查学生 对课程理论知识的掌握情况。正如前面所述, 由于学 时的限制, 教师在课堂上的讲授以理论为主, 没有时 间来讲解运筹学在实际中的应用。在考核这门课的学 习效果时, 也只能通过卷面成绩来测试学生的掌握情 况了。相对来说, 目前的考核方式比较传统, 偏应试 化。和理论类的课程考核方式一样，体现不了管理类 
课程的特点。缺乏理论在实际中的应用考核, 也缺乏 对学生运用运筹学所学优化方法来解决实际问题能 力的考核, 以及掌握软件的应用情况等。

\section{3. 运筹学课程教学创新思路}

在大数据时代和新商科背景下, 运筹学教学如何 在教学内容, 教学模式和手段等方面创新, 促进学生 学习的兴趣和动力, 调动学生学习的积极性, 增强学 生的实践学习能力, 树立正确的个人观和价值观, 做 一名合格的 21 世纪新时代的大学生, 具有重要意义。 本文针对管理类专业运筹学教学过程中存在的问题, 从以下几个方面提出些许建议供参考。

(一)、为了让学生全面认识运筹学课程, 激发 学生的学习热情, 建议在课堂教学中引入思政元素, 培养学生的价值观。运筹学起源于第二次世界大战期 间, 众多运筹学大师历经战争苦难, 对战争结束后工 农业生产过程中遇到的困难不妥协, 积极想办法增量 增产, 优化现有方案, 为全世界的经济发展做出了卓 越贡献。同样, 我国学者在运筹学领域也形成了中国 特色的研究团队 $[8,9]$ 。比如, 在 2020 年新冠肺炎疫 情爆发期间, 中国仅花了 10 天时间就在武汉建好了 火神山和雷神山两所医院, 被西方国家称为中国奇迹。 这两所医院的迅速建成离不开国家的高度重视和鼎 力支持, 更体现了建设者把运筹学优化知识运用到了 极致的思想。在建设期间, 各个班组任务如何分配, 机械设备如何调度, 工期进展如何统筹等等, 都需要 运用到运筹学的知识和相关的优化工具。在课堂教学 中, 应多结合实际生活中具有正能量的例子, 将理论 与现实结合起来, 鼓励学生学好运筹学, 用自己学到 的知识为国家和人民服务, 做一名优秀的新时代大学 生。既能调动学生学习运筹学的积极性, 又能培养学 生正确的价值观和人生观。

(二)、多引入人工智能方面的案例结合计算机 软件来教学。运筹学在实际管理决策中的应用也涉及 到很多方面, 比如表 1 就列出了运筹学在各行各业中 的应用问题。

表 1 运筹学在实际中的应用

\begin{tabular}{|c|c|}
\hline 问题类型 & 典型的问题 \\
\hline 预测 & $\begin{array}{c}\text { 对产品的需求多大, 需求类别如何, 对利 } \\
\text { 润有何影响? }\end{array}$ \\
\hline 财务 & $\begin{array}{c}\text { 需要多少资金, 从何处得到资金, 成本是 } \\
\text { 多少? }\end{array}$ \\
\hline 人力资源 & $\begin{array}{c}\text { 需要多少员工, 应具有什么技能, 留用多 } \\
\text { 长时间? }\end{array}$ \\
\hline 排序 & 什么工作最重要, 工作的顺序如何? \\
\hline 资源配置 & $\begin{array}{c}\text { 需要什么资源, 资源是否短缺, 怎样优先 } \\
\text { 获得短缺资源? }\end{array}$ \\
\hline 设备更新 & 设备运转状况如何, 可靠性如何, 什么时 \\
\hline
\end{tabular}

\begin{tabular}{|c|c|}
\hline & 候需要更新? \\
\hline 库存控制 & $\begin{array}{c}\text { 我们应保持多少库存, 什么时候应再订货, } \\
\text { 再订多少? }\end{array}$ \\
\hline 选址 & 运作的最佳场所在哪里, 需要什么设施? \\
\hline 项目规划 & $\begin{array}{c}\text { 项目需要多长时间, 哪些工作最重要, 资 } \\
\text { 源如何利用? }\end{array}$ \\
\hline 排队问题 & $\begin{array}{c}\text { 队列多长, 提供多少服务台, 能提供什么 } \\
\text { 水平的服务? }\end{array}$ \\
\hline
\end{tabular}

运筹学是数学系, 经济管理各专业以及工科相关 专业都开设的基础必须课程, 大部分教材中选取的案 例多年来没有做大的改动, 虽然经典, 但是缺乏时代 进步的特征, 尤其是体现不了大数据时代特色。建议 在实践教学中, 根据各专业的特点引入相关的现实案 例进行分析, 尤其是要找一些基于大数据, 体现人工 智能特色的运筹学案例, 与时俱进, 体现运筹学课程 的时代运用特点。比如韩国围棋九段棋手李世石和中 国围棋九段棋手柯洁，分别于 2016 年，2017 年与谷 歌公司开发的人工智能围棋程序 “阿尔法围棋”

(AlphaGo) 进行了比赛，结果都没有胜过 “阿尔法 围棋”。这就是著名的围棋人机大战。“阿尔法围棋” 程序中就运用到了大量运筹学里的动态规划方法和 决策树等知识。AlphaGo Zero 的训练流程可以分为 图 1 是所示的 4 个阶段。这里就涉及到了运筹学里动 态规划的知识。在教学过程中就可以给学生讲解这样 的例子, 让学生在学习理论知识的同时, 也能意识到 理论知识的应用性和重要性, 激发学生的学习热情, 调动学习积极性。
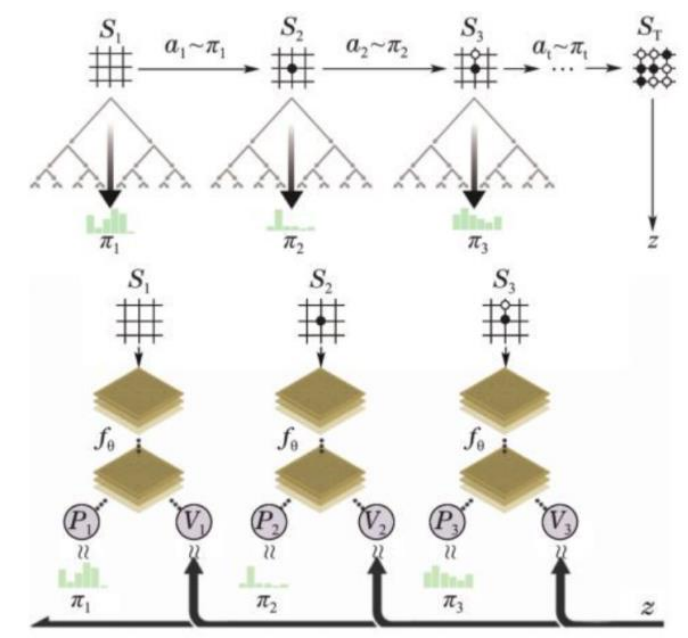

图 1 AlphaGo Zero 自我对弯流程

在教学课程中, 由于受到学时的限制, 建议适当 的轻理论, 重实践, 多引导学生在现实生活中寻找案 例。比如, 去超市观察客流量以及收银台在不同时间 段的开放情况，去公交站台统计公交车的发车间隔以 及不同时间段乘客的数量, 能否运用运筹学所学知识, 比如排队论等, 提供一个优化的解决方案等。 
(三)、翻转课堂, 鼓励学生提出问题。教师在 讲授的同时, 多鼓励学生进行思考, 在现实生活中寻 找例子, 运用所学知识来建模求解, 增强学生学习的 主动性。可以将学生分为 5 人一组, 每组利用课余时 间去生活中寻找一个相关案例, 比如, 统计超市人流 量和收银台开放的台数, 公交站点人们等待的时间和 公交车发车的间隔关系, 商场各部门上班的人数等, 进行建模分析求解, 然后在课堂上进行汇报展示。这 样的学习方式有利于促进学生学习的积极性, 进一步 了解运筹学理论知识在实际生活中的用处。让学生学 起来不至于枯燥, 同时也能更牢固地掌握理论知识。 课堂教学中, 也可以鼓励学生多参加学科竞赛, 比如 全国数学建模大赛就是一个非常合适的机会。运筹学 就是一门根据实际问题建立数学模型, 然后运用优化 方法求解, 给出方案的一门学科。通过参加比赛, 将 运筹学知识运用到比赛中, 既可以提高学习的积极性, 又可以锻炼实践能力。给学生带来学习的成就感。

(四)、制定合理的考核方案。除了平时上课考 勤, 作业和期末考试外, 可以将平时学习过程中的实 践活动纳入考核方案中。鼓励学生认真完成作业, 在 完成作业的过程中, 重点培养分析问题, 解决问题的 能力, 要注意培养实践中的问题抽象为数学模型的能 力。

比如可以将学生分组进行的案例分析作为考核 的一部分。适当减少期末考试的权重, 均匀化理论的 实践的考核比重。当前大学生对很多课程不太重视平 时的理论学习, 考前突击复习几天就能蒙混过关。如 果加大平时学习过程的考核力度, 既能促使学生认真 学好每一堂课, 又能真真正正的让学生扎扎实实的学 好运筹学这样一门课程, 对他们以后的工作生活中都 将大有禆益。

\section{4. 结论}

近年来, $5 \mathrm{G}$ 、人工智能、区块链等新兴技术正在 迅猛发展, 加上 2020 年 COVID-19 病毒在全球爆发, 人们的生活正发生着有史以来最为迅速、广泛、深刻 的变化。新的商业模式层出不穷, 大数据时代已然到 来, 对运筹学课程的教学模式和教学目标也应紧跟时 代潮流, 进行改进和创新。本文总结了当前运筹学教 学中存在的问题, 提出了教学创新的思路, 重点应进 一步加强培养学生的思维和锻炼学生的实践能力。课 程创新是一个长期积累更新的过程, 各大学应持续探 索适应新时代环境, 体现新时代特色的教学模式。适 当增加运筹学的课时, 完善实验设施, 为运筹学课程 的改革提供更好的条件。

\section{REFERENCES}

[1] Textbook Writing Group on Operations Research. (2013) Operations Research (Undergraduate Edition). Tsinghua University Press, Beijing.

[2] Hu, Y. (2018) Operations Research Tutorial.
Tsinghua University Press, Beijing.

[3] People's Daily Online. (2017) China New Business Talents Training Innovation Conference held in Shanghai.

http://finance.people.com.cn/n1/2017/1109/c100429635085.html.

[4] Guan, Y., Cui, Z.(2018) Teaching Reform of Operations Research Course Based on the Cultivation of Innovation Ability of Management Major Students. Journal of higher education, $14: 42-44$

[5] Qu, X. (2018) "Flipped Classroom" in Operations Research Teaching under the Background of "Internet +". Journal of Heilongjiang Institute of Education, 37 (7): 52-54.

[6] Zou, Q., Chen J. (2013) Practical Experience of Operations Research Software in the Teaching of Economic Management Operations Research. University Education, 22: 60-62.

[7] Ma, M., Liu, J. (2020) Research on Curriculum Ideology and Politics in Operations Research Curriculum Teaching. Journal of Higher Education, 157(35): 182-185.

[8] Chen, H., Wang, S. (2019) Teaching Reform and Practice of Operations Research Course. Education and Teaching Forum, 42: 125-126.

[9] Li, Z., Zhang, X., Liu, N. (2020) Research on the Teaching Reform of Operations Research Curriculum based on the Perspective of Management. Education Modernization, 9: 38-40. 